\title{
Image restoration with a half-quadratic approach to mixed weighted smooth and anisotropic bounded variation regularization
}

\author{
Francisco Javier Ibarrola ${ }^{1}$, Ruben Daniel Spies ${ }^{1,2 *}$ \\ ${ }^{1}$ Departamento de Matemática, Facultad de Ingeniería Química, Universidad Nacional del Litoral, Santiago del Estero 2829, \\ S3000AOM Santa Fe, Argentina. \\ ${ }^{2}$ Instituto de Matemática Aplicada del Litoral, IMAL, CONICET-UNL, Centro Científico Tecnológico CONICET Santa Fe, \\ Colectora Ruta Nac. $N^{\circ} 168$ km. 472, Paraje "El Pozo”, 3000 Santa Fe, Argentina. \\ *Corresponding author: rspies@santafe-conicet.gov.ar
}

\begin{abstract}
:
The problem of restoring a signal or image is often tantamount to approximating the solution of a linear inverse ill-posed problem. In order to find such an approximation one might regularize the problem by penalizing variations on the estimated solution. Among the wide variety of methods available to perform this penalization, the most commonly used is the Tikhonov-Phillips regularization, which is appropriate when the sought signal or image is expected to be smooth, but it results unsuitable whenever preservation of discontinuities and edges is an important matter. Nonetheless, there are other methods with edge preserving properties, such as bounded variation $(B V)$ regularization. However, these methods tend to produce piecewise constant solutions showing the so called "staircasing effect" and their numerical implementations entail great computational effort and cost. In order to overcome these obstacles, we consider a mixed weighted Tikhonov and anisotropic $B V$ regularization method to obtain improved restorations and we use a half-quadratic approach to construct highly efficient numerical algorithms. Several numerical results in signal and image restoration problems are presented.
\end{abstract}

Keywords:

Inverse Problems; III-posedness; Regularization; Half-Quadratic

\section{Introduction and preliminaries}

In a general context, a linear inverse problem can be formulated as the need of finding $w$ in an equation of the form

$$
\mathscr{T} w=y,
$$

where $\mathscr{T}: \mathscr{X} \rightarrow \mathscr{Y}$ is a bounded linear operator between two infinite dimensional Hilbert spaces $\mathscr{X}$ and $\mathscr{Y}$, the range of $\mathscr{T}$ is non-closed and $y$ is the data, which is supposed to be known, perhaps with a certain degree of error. In the sequel and unless otherwise specified, the space $\mathscr{X}$ will be $L^{2}(\Omega)$ where $\Omega \subset \mathbb{R}^{n}$ 
is a bounded open convex set with Lipschitz boundary. Under these hypotheses, it turns out that problem (1) is ill-posed in the sense of Hadamard ([1]), the Moore-Penrose inverse of $\mathscr{T}$ is unbounded and small errors in the data $y$ may result in very large errors in the corresponding approximations of $w$ ([2]). Before any attempt is made to approximate the solution of (1), the problem must be "regularized". Regularizing an inverse problem consists essentially of replacing the problem by a sequence of "well-posed" problems whose solutions converge (in an appropriate way) to a solution (or to a least squares solution) of (1). Using the Tikhonov-Phillips method is undoubtedly the most common way of regularizing an ill-posed problem. Although the method can be formulated within a very general mathematical theory by means of spectral theory (see [3]), the widespread of its use is mainly due to the fact that it can also be formulated as an unconstrained optimization problem. In fact, given an appropriate functional $P(w)$ (in the sequel we shall refer to $P$ as a penalizer) with domain $\mathscr{D} \subset \mathscr{X}$, the regularized solution obtained by the Tikhonov-Phillips method and such a penalizer, is the global minimizer $w_{\alpha}$ (provided it exists), over $\mathscr{D}$, of the functional

$$
J_{\alpha, P}(w)=\|\mathscr{T} w-y\|^{2}+\alpha P(w),
$$

where $\alpha$ is a positive constant called regularization parameter. The original method was proposed independently by Phillips and Tikhonov in 1962 and 1963 ([4], [5]) using $P(w)=\|w\|_{L^{2}(\Omega)}^{2}$. Other penalizers can also be used to regularize the problem. Each choice of $P$ results in a different regularized solution possessing particular properties. In the past 15 years considerable attention has been given to finding "appropriate" penalizers for a given problem. Thus, for instance, it is well known that the choice of $P(w)=\|w\|_{L^{2}(\Omega)}^{2}$ produces always smooth regularized approximations which converge, as $\alpha \rightarrow 0^{+}$, to the best approximate solution (i.e. the least squares solution of minimum norm) of problem (1) (see [3]) while for $P(w)=\||\nabla w|\|_{L^{2}(\Omega)}^{2}$ the order-one Tikhonov-Phillips method is obtained. Similarly, the choice of $P(w)=\|w\|_{\mathrm{BV}(\Omega)}$ (where $\|\cdot\|_{\mathrm{BV}}$ denotes the total variation norm) or $P(w)=\||\nabla w|\|_{L^{1}(\Omega)}$, result in the so called "bounded variation regularization methods" ([6], [7]). The use of these of penalizers is strongly suggested when preserving discontinuities or edges is an important matter. The method, however, tends to produce piecewise constant approximations and therefore it will most likely be inappropriate in regions where the exact solution is smooth ([8]), producing the so called "staircasing effect". For general penalizers $P$, sufficient conditions guaranteeing existence, uniqueness and weak and strong stability of the minimizers under different types of perturbations were found in [9].

Given that each penalizing term engraves the solution with particular properties, in certain types of problems, particularly in those in which it is known that the regularity of the exact solution is heterogeneous and/or anisotropic, it is reasonable to think that the use of two or more penalizers of different nature, that could somehow spatially adapt to the local characteristics of the exact solution, would be more convenient. During the last 15 years many regularization methods have been developed in light of this simple reasoning. Thus, for instance, in 1997 Blomgren et al. ([10]) proposed the following penalizer:

$$
P(w)=\int_{\Omega}|\nabla w|^{p(|\nabla w|)} d x
$$

where $p$ is a decreasing function satisfying $\lim _{u \rightarrow 0^{+}} p(u)=2, \lim _{u \rightarrow \infty} p(u)=1$. Thus, in regions where the gradient of $w$ is small the penalizer is approximately equal to $\||\nabla w|\|_{L^{2}(\Omega)}^{2}$, corresponding to a TikhonovPhillips method of order one (appropriate for smooth regions). On the other hand, when the modulus of the gradient of $w$ is large, the penalizer resembles the bounded variation seminorm $\||\nabla w|\|_{L^{1}(\Omega)}$, which, as previously mentioned, is a good choice for border detection purposes. Although this model for $P$ is quite reasonable, proving basic properties of the corresponding generalized Tikhonov-Phillips functional turns out to be quite difficult. The authors proved existence of global minimizers of functional (2), by using the 
theory of variable $L^{p}$ spaces. In 1997 Chambolle and Lions suggested a different way of combining these two methods ([8]). They defined a thresholded penalizer of the form:

$$
P_{\beta}(w)=\int_{|\nabla w| \leq \beta}|\nabla w|^{2} d x+\int_{|\nabla w|>\beta}|\nabla w| d x
$$

where $\beta>0$ is a prescribed threshold parameter. Thus, in regions where borders are more likely to be present $(|\nabla w|>\beta)$, penalization is made with the bounded variation seminorm while a standard order-one Tikhonov-Phillips method is used otherwise. This model was shown to be successful in restoring images possessing regions with homogeneous intensity separated by borders. However, in the case of images with non-uniform or highly degraded intensities, the model is extremely sensitive to the choice of the threshold parameter $\beta$. More recently, penalizers of the form

$$
P(w)=\int_{\Omega}|\nabla w|^{p(x)} d x
$$

for certain functions $p$ with range in [1,2], were studied in [11] and [12]. It is timely to point out here that all previously mentioned results are valid only for the case of denoising (no blurring), i.e. for the case $\mathscr{T}=i d$. More recently, Mazzieri, Spies and Temperini studied penalizers of the form

$$
P(w)=\lambda_{0} \int_{\Omega}|\sqrt{1-\theta(x)} w(x)|^{2} d x+\lambda_{1} \int_{\Omega}\|\theta(x) \mathscr{A}(x) \nabla w(x)\| d x
$$

where $\lambda_{0}, \lambda_{1}$ are positive constants, $\theta(x)$ is a weighting function with values on the interval $[0,1]$ and $A(x)$ is a symmetric positive-definite matrix field, introduced to take into account possible local anisotropic penalization. General existence, uniqueness and stability results of global minimizers of the corresponding generalized Tikhonov-Phillips functionals

$$
\mathscr{J}_{\theta}(w)=\|\mathscr{T} w-y\|^{2}+\lambda_{0} \int_{\Omega}|\sqrt{1-\theta(x)} w(x)|^{2} d x+\lambda_{1} \int_{\Omega}\|\theta(x) \mathscr{A}(x) \nabla w(x)\| d x
$$

can be found in [13] and [14]. Several remarks are in order. First note that the extreme case $\theta(x)=0 \forall x$ corresponds to the classical Tikhonov-Phillips method. For $\theta(x)=1 \forall x$ one gets a pure $B V$ method, with the classical Bounded Variation method corresponding to the case of $\mathscr{A}(x)=i d \forall x$. Other choices of the matrix field $A$ are possible in order to induce anisotropic $B V$ penalization. Feasible ways of constructing this matrix field can be found for instance in [15]. The general case can then be thought of as a convex combination of classical $L^{2}$ and anisotropic $B V$ penalizers.

\section{Signal restoration with a half quadratic approach to mixed regulariza- tion}

Approximating the minimizer of (6) presents quite serious computational challenges. In fact, for high dimensional problems like those arising in image restoration, all standard optimization algorithms will require many hours of CPU time in any modern personal computer. In order to reduce the computational burden originated by this difficulty, we shall next consider an alternative approach to the problem of finding the minimizer of (6). The tactic is based upon the general ideas introduced by D. Geman in [16] for image recovery and used later by J. Idier in [17] for solving detection-estimation problems, and it consists of rewriting the functional in an appropriate form via half-quadratic optimization tools. To 
introduce this approach, we will first consider the case of minimizing functional (6) when $\Omega$ is a subset of $\mathbb{R}$, meaning $w(x)$ represents the signal we wish to restore. Without loss of generality we shall assume $\Omega=[0,1]$. The operator $\mathscr{A}$ will necessarily be the identity, meaning our functional takes the form

$$
\mathscr{J}_{\theta}(w)=\|\mathscr{T} w-y\|^{2}+\lambda_{0} \int_{0}^{1}|\sqrt{1-\theta(x)} w(x)|^{2} d x+\lambda_{1} \int_{0}^{1}\left|\theta(x) w^{\prime}(x)\right| d x .
$$

\subsection{Signal restoration by mixed regularization}

Since restoring the signal $w(x)$ is tantamount to finding the minimizer of the previous functional, in order to proceed numerically we will need to discretize the problem. For that, we will take $M$ equally spaced points $x_{m} \in[0,1]$ and define our discretized signal $u \in \mathbb{R}^{M}$ by $u_{m} \doteq w\left(x_{m}\right)$ for $m=1,2, \ldots, M$. In the same way, let $T \in \mathbb{R}^{N \times M}$ and $v \in \mathbb{R}^{N}$ be discretized versions of the operator $\mathscr{T}$ and of the observation $y$, respectively, and let $\theta_{m} \doteq \theta\left(x_{m}\right)$ for $m=1,2, \ldots, M$. We now introduce a discrete finite-differences approximation of functional ( 7 ) as follows:

$$
J_{\theta}(u)=\|T u-v\|^{2}+\frac{\lambda_{0}}{M} \sum_{m=1}^{M}\left(1-\theta_{m}\right) u_{m}^{2}+\frac{\lambda_{1}}{M} \sum_{m=2}^{M} \theta_{m}\left|\frac{u_{m}-u_{m-1}}{1 / M}\right|
$$

The restored signal $w(x)$ will then be approximated by the discrete signal represented by the vector minimizing this functional. The main difficulty for finding such a minimizer arises from the nondifferentiability of the absolute value at the origin, which precludes the differentiability of $J_{\theta}(u)$. To overcome this impediment we shall replace the absolute value by a function $\phi(t)$ approximating it and satisfying certain additional regularity and asymptotic assumptions. For general non-quadratic penalizers, in order to preserve edges and discontinuities between homogeneous regions, it is important to appropriately choose the behavior of this function $\phi$. Two groups of functions have mainly been considered in the literature. Namely:

- $L_{2} L_{1}$ : we say that a function $\phi: \mathbb{R} \rightarrow \mathbb{R}$ is of $L_{2} L_{1}$ class if it is even, non-constant, convex and of class $\mathscr{C}^{1}$ on $\mathbb{R}, \mathscr{C}^{2}$ at the origin and asymptotically linear.

- $L_{2} L_{0}$ : we say that a function $\phi: \mathbb{R} \rightarrow \mathbb{R}$ is of $L_{2} L_{0}$ class if it is even, non-constant, non-decreasing on $\mathbb{R}^{+}$, asymptotically constant and of class $\mathscr{C}^{2}$ at the origin.

It is important to point out that an $L_{2} L_{1}$ or $L_{2} L_{0}$ function does not need to be an approximation of the absolute value. Thus, for instance, in certain detection-estimation problems for the case of restoration of piecewise smooth signals, the $L_{2} L_{0}$ function $\phi(t)=\min \left\{t^{2}, \eta^{2}\right\}$ (for a given $\eta$ ), results appropriate ([17], Section 6.4.1). In our case, we will pick the $L_{2} L_{1}$ function

$$
\phi(t)=\phi_{\eta}(t) \doteq \sqrt{t^{2}+\eta^{2}}-\eta
$$

for $\eta>0$ sufficiently small, and replace functional (8) with

$$
J_{\theta, \phi}(u)=\|T u-v\|^{2}+\frac{\lambda_{0}}{M} \sum_{m=1}^{M}\left(1-\theta_{m}\right) u_{m}^{2}+\frac{\lambda_{1}}{M} \sum_{m=2}^{M} \theta_{m} \phi\left(\frac{u_{m}-u_{m-1}}{1 / M}\right) .
$$

Now that we have a differentiable functional, we will introduce a duality relation which will later allow us to conveniently write the corresponding first order necessary condition as a linear system. 


\subsection{Non-quadratic and half-quadratic duality}

Let $\phi(t)$ be a non-quadratic function satisfying: (i) $\phi(t)$ is even; (ii) $\phi(\sqrt{t})$ is concave on $\mathbb{R}^{+}$and (iii) $\phi(t)$ is continuous at $t=0$ and $\phi \in \mathscr{C}^{1}(\mathbb{R} \backslash\{0\})$. Under these hypotheses, it can be shown ([18], Section $12)$ that there exists a function $\psi(b)$ so that the pair $(\phi, \psi)$ satisfy the following duality relation:

$$
\begin{gathered}
\phi(t)=\inf _{s>0}\left(s t^{2}+\psi(s)\right), \\
\psi(s)=\sup _{t \in \mathbb{R}}\left(\phi(t)-s t^{2}\right) .
\end{gathered}
$$

Expression (11) is usually referred to as the half-quadratic form of $\phi$. For instance, for $\phi=\phi_{\eta}$ as in (9), it can be easily shown that the corresponding dual function is

$$
\psi_{\eta}(s)=\eta^{2} s-\eta+\frac{1}{4 s}
$$

By using the dual function $\psi(s)$ we now define the following functional, which introduces an auxiliary variable $s \in \mathbb{R}_{+}^{M-1}$ :

$$
K_{\theta, \phi}(u, s) \doteq\|T u-v\|^{2}+\frac{\lambda_{0}}{M} \sum_{m=1}^{M}\left(1-\theta_{m}\right) u_{m}^{2}+\frac{\lambda_{1}}{M} \sum_{m=2}^{M} \theta_{m}\left(s_{m}\left(\frac{u_{m}-u_{m-1}}{1 / M}\right)^{2}+\psi\left(s_{m}\right)\right) .
$$

Now, equations (10) and (13) are closely related. In fact, by minimizing $K_{\theta, \phi}(u, s)$ with respect to $s \in \mathbb{R}_{+}^{M-1}$ we obtain:

$$
\begin{aligned}
& \inf _{s \in \mathbb{R}_{+}^{M-1}} K_{\theta, \phi}(u, s) \\
= & \|T u-v\|^{2}+\frac{\lambda_{0}}{M} \sum_{m=1}^{M}\left(1-\theta_{m}\right) u_{m}^{2}+\frac{\lambda_{1}}{M} \inf _{s \in \mathbb{R}_{+}^{M-1}}\left[\sum_{m=2}^{M} \theta_{m}\left(s_{m}\left(\frac{u_{m}-u_{m-1}}{1 / M}\right)^{2}+\psi\left(s_{m}\right)\right)\right] \\
= & \|T u-v\|^{2}+\frac{\lambda_{0}}{M} \sum_{m=1}^{M}\left(1-\theta_{m}\right) u_{m}^{2}+\frac{\lambda_{1}}{M} \sum_{m=2}^{M} \theta_{m} \inf _{s_{m} \in \mathbb{R}_{+}}\left(s_{m}\left(\frac{u_{m}-u_{m-1}}{1 / M}\right)^{2}+\psi\left(s_{m}\right)\right) \\
= & \|T u-v\|^{2}+\frac{\lambda_{0}}{M} \sum_{m=1}^{M}\left(1-\theta_{m}\right) u_{m}^{2}+\frac{\lambda_{1}}{M} \sum_{m=2}^{M} \theta_{m} \phi\left(\frac{u_{m}-u_{m-1}}{1 / M}\right) \\
= & J_{\theta, \phi}(u),
\end{aligned}
$$

where the second equality holds since each element of $s$ is associated independently to one and only one term of the sum. Our goal can then be thought of as minimizing $K_{\theta, \phi}(u, s)$ with respect to both $u$ and $s$. We start by defining a diagonal matrix $S \in \mathbb{R}^{M \times M}$, with diagonal elements $s_{m}$ such that $s_{1}=0$, and $s_{m}$ are the components of vector $s$. Furthermore, by defining the diagonal matrix $\Theta \in \mathbb{R}^{M \times M}$ by $\Theta_{m, m}=\theta_{m}$ and letting $L_{1}$ be the one-dimensional first order finite difference matrix (i.e. $\left(L_{1} u\right)_{m}=u_{m}-u_{m-1}$ for $m=2,3, \ldots, M$ and $L_{1,1}=0$ ), we can finally write

$$
K_{\theta, \phi}(u, s)=\|T u-v\|^{2}+\frac{\lambda_{0}}{M} u^{t}\left(I_{M}-\Theta\right) u+\lambda_{1} M u^{t} L_{1}^{t} \Theta S L_{1} u+\frac{\lambda_{1}}{M} \sum_{m=2}^{M} \theta_{m} \psi\left(s_{m}\right) .
$$


The minimizer of $K_{\theta, \phi}(u, s)$ with respect to $u$ can be found by simply solving the system given by the first order necessary condition, which turns out to be linear. In fact, from expression (15) we obtain

$$
\left(T^{t} T+\frac{\lambda_{0}}{M}\left(I_{M}-\Theta\right)+\lambda_{1} M L_{1}^{t} \Theta S L_{1}\right) u=T^{t} v
$$

Nonetheless, we still need to minimize $K_{\theta, \phi}(u, s)$ with respect to $s$. Here again, the duality relation comes in handy since by differentiating expression (11) we observe that the elements $b_{m} \doteq$ $\underset{s_{m} \in \mathbb{R}_{+}}{\arg \min }\left(s_{m}\left(\frac{u_{m}-u_{m-1}}{1 / M}\right)^{2}+\psi\left(s_{m}\right)\right)$ must satisfy

$$
b_{m}=\frac{\phi^{\prime}\left(t_{m}\right)}{2 t_{m}}
$$

where $t_{m}=\left(u_{m}-u_{m-1}\right) /(1 / M)$. Let us notice that $\phi(t)$ has continuous second order derivative at zero, provided that $\phi(t)$ be of class $L_{2} L_{1}$ or $L_{2} L_{0}$, and then L'Hôpital's rule implies that $\frac{\phi^{\prime}(t)}{2 t}$ has a removable singularity at zero. Hence for $t_{m}=0$, we define $b_{m}=\phi^{\prime \prime}(0) / 2$.

We shall now proceed to build an algorithm for approximating the minimizer of $J_{\theta, \phi}(u)$ by using expressions (16) and (17) via a fixed-point type argument as follows.

\subsection{Numerical implementation of the half-quadratic approach}

Since minimizing (15) implies the simultaneous minimization with respect to the inter-dependent variables $u$ and $s$, we will state a simple cyclic iterative algorithm that was found to be quite effective:

Step 1 - Initializing: set $j=0$, and initialize $u^{j}=u^{0}$.

Step 2 - Counting: let $j=j+1$.

Step 3 - Updating $\mathbf{b}_{\mathbf{m}}$ : update $b_{m}^{j}$ using equation (17). That is,

$$
b_{m}^{j} \doteq \frac{\phi^{\prime}\left(M\left(u_{m}^{j-1}-u_{m-1}^{j-1}\right)\right)}{2 M\left(u_{m}^{j-1}-u_{m-1}^{j-1}\right)}, \quad m=2, \ldots, M
$$

Step 4 - Updating u: update $u^{j}$ by solving (in the least-squares sense) the linear system

$$
\left(T^{t} T+\frac{\lambda_{0}}{M}\left(I_{M}-\Theta\right)+\lambda_{1} M \Theta L_{1}^{t} B^{j} L_{1}\right) u^{j}=T^{t} v
$$

where $B^{j}$ is $M$-by- $M$ diagonal matrix with its first diagonal element 0 followed by $b_{2}^{j}, b_{3}^{j}, \ldots, b_{M}^{j}$.

Step 5 - Convergence: if a previously defined convergence criterion is satisfied, the algorithm ends and $u^{j}$ is taken as the restored signal. Else, the algorithm repeats from step 2.

In the next section, we shall generalize this half-quadratic approach to the case of image restoration problems (i.e. for $n=2$ ). 


\section{The half-quadratic approach to image restoration with mixed $\mathbf{L}^{2}$ and anisotropic BV regularization}

Consider now the model problem (1) along with functional (6) and assume $\Omega=[0,1] \times[0,1]$. Here now $w(x)$ represents the intensity of a gray-scale image at the point $x \in \Omega$. We discretize the image to obtain an $M$-by- $M$ matrix $U$, consisting of the values of $w$ at the centerpoints of an $M$-by- $M$ pixel grid. Next, we stack the columns of $U$ to get a vector $u \in \mathbb{R}^{M^{2}}$ so that $u_{M(l-1)+m}=U_{m, l} \forall l, m=1,2, \ldots, M$. We proceed in the same way to obtain $\Theta \in \mathbb{R}^{M^{2}}$ and $v \in \mathbb{R}^{N^{2}}$, corresponding to discretized versions of $\theta(\cdot)$ and of the observation $y$, respectively. Finally, $T \in \mathbb{R}^{N^{2} \times M^{2}}$ will represent an appropriately discretized version of the operator $\mathscr{T}$ and $A_{m}$ the 2-by-2 matrix obtained by evaluating the matrix-valued function $\mathscr{A}(\cdot)$ at the centerpoint of the $m^{\text {th }}$ pixel. Thus, our discretization of functional (6) takes the form

$$
J_{\theta}(u)=\|T u-v\|^{2}+\frac{\lambda_{0}}{M^{2}} \sum_{m=1}^{M^{2}}\left(1-\theta_{m}\right) u_{m}^{2}+\frac{\lambda_{1}}{M^{2}} \sum_{m \in \mathfrak{M}} \theta_{m}\left\|A_{m}\left(\begin{array}{c}
M\left(u_{m}-u_{m+1}\right) \\
M\left(u_{m}-u_{m-M}\right)
\end{array}\right)\right\|_{1},
$$

where $\mathfrak{M}$ is the set of all indexes which do not correspond to a pixel in the bottom or left borders of the image. Naturally, the restored image will be approximated by the minimizer of this functional. In order to find such a minimizer, let us begin by noticing that the $m$-th term of the discretized anisotropy penalizer on (18) is now

$$
\begin{aligned}
\left\|A_{m}\left(\begin{array}{c}
M\left(u_{m}-u_{m+1}\right) \\
M\left(u_{m}-u_{m-M}\right)
\end{array}\right)\right\|_{1} & =\left|M\left(a_{1,1}^{m}\left(u_{m}-u_{m-M}\right)+a_{1,2}^{m}\left(u_{m}-u_{m+1}\right)\right)\right| \\
& +\left|M\left(a_{2,1}^{m}\left(u_{m}-u_{m-M}\right)+a_{2,2}^{m}\left(u_{m}-u_{m+1}\right)\right)\right|,
\end{aligned}
$$

where $a_{i, j}^{m}, i, j=1,2$, are the elements of the 2 -by-2 matrix $A_{m}$. Here again, to avoid the nondifferentiability of the absolute value at the origin, we will replace it with the function $\phi(t)=\phi_{\eta}(t)$ defined in (9), for which the corresponding dual function is $\psi(s)=\psi_{\eta}(s)$ given by (12). Following the same steps as in section 2.2 and using the duality relation (11), we approximate (19) by

$$
\begin{aligned}
\left\|A_{m}\left(\begin{array}{c}
M\left(u_{m}-u_{m+1}\right) \\
M\left(u_{m}-u_{m-M}\right)
\end{array}\right)\right\|_{1} & \approx \inf _{s \in \mathbb{R}^{M^{2}}}\left(s_{m}\left[M\left(a_{1,1}^{m}\left(u_{m}-u_{m-M}\right)+a_{1,2}^{m}\left(u_{m}-u_{m+1}\right)\right)\right]^{2}+\psi\left(s_{m}\right)\right) \\
& +\inf _{q \in \mathbb{R}^{M^{2}}}\left(q_{m}\left[M\left(a_{2,1}^{m}\left(u_{m}-u_{m-M}\right)+a_{2,2}^{m}\left(u_{m}-u_{m+1}\right)\right)\right]^{2}+\psi\left(q_{m}\right)\right),
\end{aligned}
$$

Define now the $M^{2}$-by- $M^{2}$ diagonal matrices $A^{i, j}$ for $i, j=1,2$, such that $A_{m, m}^{i, j}=a_{i, j}^{m}$ if $m \in \mathfrak{M}$ and $A_{m, m}^{i, j}=$ 0 otherwise, and let $R_{1}$ and $R_{2}$ be the $M^{2}$-by- $M^{2}$ matrices defined by $R_{1} \doteq A^{1,1}\left(L_{1} \otimes I_{M}\right)+A^{1,2}\left(I_{M} \otimes L_{1}^{t}\right)$ and $R_{2} \doteq A^{2,1}\left(L_{1} \otimes I_{M}\right)+A^{2,2}\left(I_{M} \otimes L_{1}^{t}\right)$, where $I_{M}$ denotes the $M$-by- $M$ indentity matrix and $\otimes$ is the Kronecker product. We then build, in the same way we did for signals, the functional

$$
\begin{aligned}
K_{\theta, \phi}(u, s, q)= & \|T u-v\|^{2}+\frac{\lambda_{0}}{M^{2}} u^{t}\left(I_{M^{2}}-\Theta\right) u+\lambda_{1} u^{t} R_{1}^{t} \Theta S R_{1} u+\lambda_{1} u^{t} R_{2}^{t} \Theta Q R_{2} u \\
& +\frac{\lambda_{1}}{M^{2}} \sum \theta_{m} \psi\left(s_{m}\right)+\frac{\lambda_{1}}{M^{2}} \sum \theta_{m} \psi\left(r_{m}\right),
\end{aligned}
$$

where $S$ and $Q$ are the $M^{2}$-by- $M^{2}$ diagonal matrices whose diagonal elements are $s_{m}$ and $q_{m}$, respectively. It can now be easily seen that

$$
\inf _{s, q \in \mathbb{R}_{+}^{M^{2}}} K_{\theta, \phi}(u, s, q)=J_{\theta, \psi}(u) \approx J_{\theta}(u)
$$


where $J_{\theta, \psi}(u)$ is the approximation of (18) obtained by using (20). We now want to find the minimizer of functional (21) with respect to $u$. As for the case of signals (equation (15)), the first order necessary condition of $K_{\theta, \phi}(u, s, q)$ with respecto to $u$ results in a linear system, given by

$$
\left(T^{t} T+\frac{\lambda_{0}}{M^{2}}\left(I_{M^{2}}-\Theta\right)+\lambda_{1} R_{1}^{t} \Theta S R_{1}+\lambda_{1} R_{2}^{t} \Theta Q R_{2}\right) u=T^{t} v
$$

Since we also need to minimize $K_{\theta, \phi}(u, s, q)$ with respect to $s$ and $q$, we define

$$
b_{m} \doteq \underset{s_{m} \in \mathbb{R}_{+}}{\arg \min }\left(s_{m} M^{2}\left(a_{1,1}^{m}\left(u_{m}-u_{m-M}\right)+a_{1,2}^{m}\left(u_{m}-u_{m+1}\right)\right)^{2}+\psi\left(s_{m}\right)\right) \text {, }
$$

and

$$
c_{m} \doteq \underset{q_{m} \in \mathbb{R}_{+}}{\arg \min }\left(q_{m} M^{2}\left(a_{2,1}^{m}\left(u_{m}-u_{m-M}\right)+a_{2,2}^{m}\left(u_{m}-u_{m+1}\right)\right)^{2}+\psi\left(q_{m}\right)\right) .
$$

We now resort to (11) to deduce that the elements $b_{m}$ and $c_{m}$ must satisfy

$$
b_{m}=\frac{\phi^{\prime}\left(M\left(a_{1,1}^{m}\left(u_{m}-u_{m-M}\right)+a_{1,2}^{m}\left(u_{m}-u_{m+1}\right)\right)\right)}{2 M\left(a_{1,1}^{m}\left(u_{m}-u_{m-M}\right)+a_{1,2}^{m}\left(u_{m}-u_{m+1}\right)\right)}
$$

and

$$
c_{m}=\frac{\phi^{\prime}\left(M\left(a_{2,1}^{m}\left(u_{m}-u_{m-M}\right)+a_{2,2}^{m}\left(u_{m}-u_{m+1}\right)\right)\right)}{2 M\left(a_{2,1}^{m}\left(u_{m}-u_{m-M}\right)+a_{2,2}^{m}\left(u_{m}-u_{m+1}\right)\right)} .
$$

Based upon all of the above, we shall state a cyclic iterative algorithm for image restoration as follows:

Step 1 - Initializing: set $j=0$, and initialize $u^{j}=u^{0}$.

Step 2 - Counting: let $j=j+1$.

Step 3 - Updating $\mathbf{b}_{\mathbf{m}}^{\mathbf{j}}$ : update $b_{m}^{j}$ using equation (24). That is,

$$
b_{m}^{j}=\frac{\phi^{\prime}\left(M\left(a_{1,1}^{m}\left(u_{m}^{j-1}-u_{m-M}^{j-1}\right)+a_{1,2}^{m}\left(u_{m}^{j-1}-u_{m+1}^{j-1}\right)\right)\right)}{2 M\left(a_{1,1}^{m}\left(u_{m}^{j-1}-u_{m-M}^{j-1}\right)+a_{1,2}^{m}\left(u_{m}^{j-1}-u_{m+1}^{j-1}\right)\right)}
$$

Step 4 - Updating $\mathbf{c}_{\mathbf{m}}^{\mathbf{j}}$ : update $c_{m}^{j}$ using equation (25). That is,

$$
c_{m}^{j}=\frac{\phi^{\prime}\left(M\left(a_{2,1}^{m}\left(u_{m}^{j-1}-u_{m-M}^{j-1}\right)+a_{2,2}^{m}\left(u_{m}^{j-1}-u_{m+1}^{j-1}\right)\right)\right)}{2 M\left(a_{2,1}^{m}\left(u_{m}^{j-1}-u_{m-M}^{j-1}\right)+a_{2,2}^{m}\left(u_{m}^{j-1}-u_{m+1}^{j-1}\right)\right)}
$$

Step 5 - Updating u: update $u^{j}$ by solving the linear system

$$
\left(T^{t} T+\frac{\lambda_{0}}{M^{2}}\left(I_{M^{2}}-\Theta\right)+\lambda_{1} R_{1}^{t} \Theta B^{j} R_{1}+\lambda_{1} R_{2}^{t} \Theta C^{j} R_{2}\right) u^{j}=T^{t} v
$$

where $B^{j}$ and $C^{j}$ are the $M^{2}$-by- $M^{2}$ diagonal matrices with elements $b_{m}^{j}$ and $c_{m}^{j}$ for $m \in \mathfrak{M}$ and 0 otherwise.

Step 6 - Convergence: if a previously defined convergency criterion is satisfied, the algorithm ends and our restored signal is defined as $u^{j}$. Else, the algorithm repeats from step 2. 


\section{Applications to signal and image restoration}

The purpose of this section is to present some applications of the algorithms developed above for half-quadratic mixed $L^{2}-B V$ regularization in signal and image restoration problems.

\subsection{A signal restoration example}

A basic mathematical model for signal blurring is given by convolution as a Fredholm integral equation of first kind:

$$
y(x)=\int_{0}^{1} k(t, x) w(t) d t \doteq \mathscr{T} w(x),
$$

where $w$ and $y$ are the original and blurred signal, respectively, and $k(t, x)$ is called point spread function (PSF) or blurring kernel. For the numerical examples that follow we took $k(t, x)$ to be Gaussian, i.e. $k(t, x)=\frac{1}{\sqrt{2 \pi} \sigma_{b}} \exp \left(-\frac{(t-x)^{2}}{2 \sigma_{b}^{2}}\right)$, with $\sigma_{b}>0$ and equation (26) was discretized in the usual way (using collocation and quadrature), resulting in a discrete model of the form

$$
T u=v,
$$

where $T$ is an $(M+1) \times(M+1)$ matrix and $u, v \in \mathbb{R}^{M+1}\left(u_{j}=w\left(x_{j}\right), v_{j}=y\left(x_{j}\right), x_{j}=\frac{j}{M}, 0 \leq j \leq M\right)$. We took $M=130$ and $\sigma_{b}=0.04$. The data $g$ was contaminated with a $1.5 \%$ zero-mean Gaussian additive noise (i.e. standard deviation equal to $1.5 \%$ of the range of $v$ ). Figure 1(a) shows the original signal $u$ (unknown in real life problems) and the blurred noisy signal $v$ which constitutes the data of the inverse problem. Figure 1(b) shows the restoration obtained with the classical Tikhonov-Phillips method (corresponding to $\theta(x)=0$ in functional (6) ). Figure 1(c) shows the restoration obtained with the pure $B V$ penalizer $(\theta(x)=1$ in (6) ) and finally, Figure 1(d) depicts the restoration obtained with the mixed $L^{2}-B V$ penalizer with $\theta(x)$ chosen by scaling to $[0,1]$ the absolute value of the derivative of the regularized solution obtained with a pure Tikhonov-Phillips method. It is timely to point out here that the choice of $\theta$ is a very important matter about which we shall not discuss in this article. In all cases, the regularization parameters used were estimated using Morozov's discrepancy principle. 
(a)

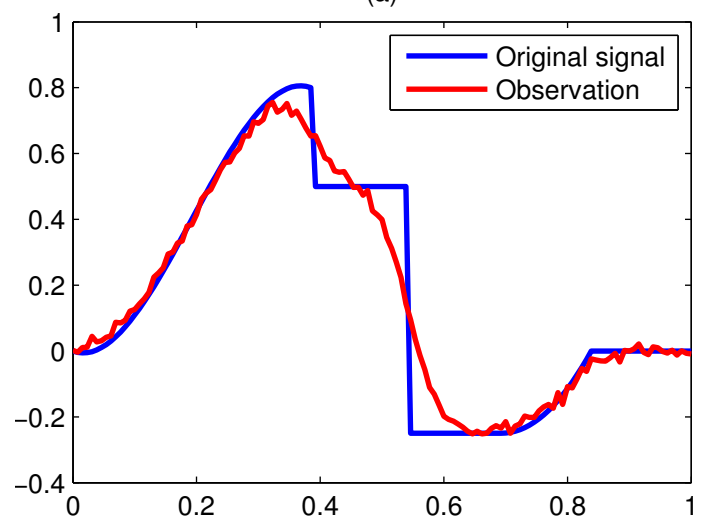

(c)

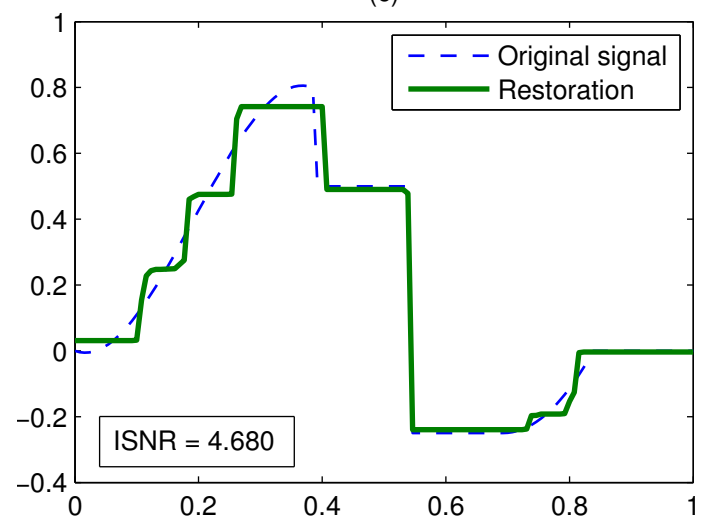

(b)

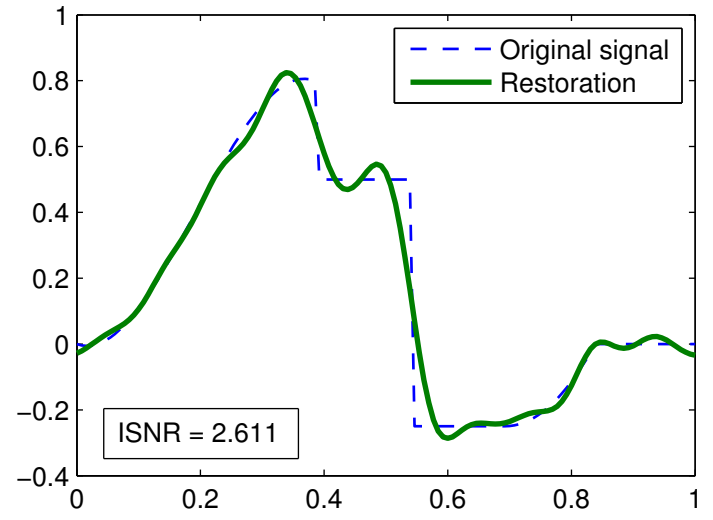

(d)

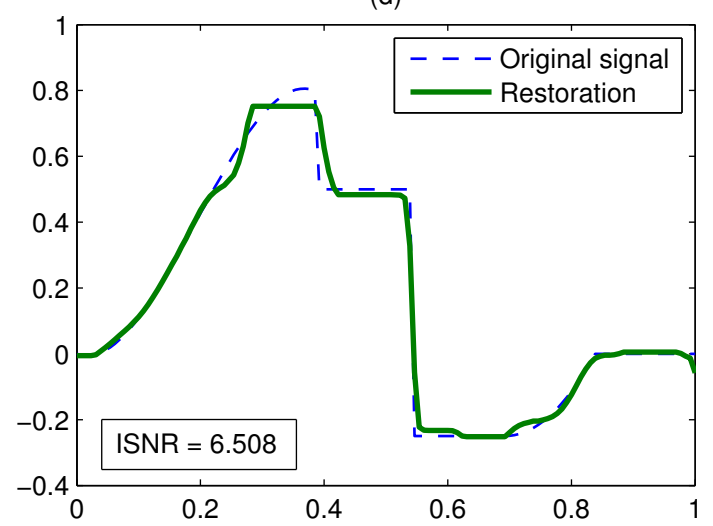

Figure 1: (a) Original signal and observation; (b) Tikhonov-Phillips restoration; (c) $B V$ restoration; (d) Mixed $L^{2}-\mathrm{BV}$ restoration.

As expected, the combined method returns a much better restoration. This is clearly reflected in the ISNR values for all three cases. The CPU times required to perform the latter mixed restoration was compared to those required by another program based in the traditional Newton-Raphson method. Given the same stopping criteria, the algorithm proposed here was in average over thirty five times faster than the other, what provides strong evidence of the computational efficiency of these new half-quadratic approach. We will next present a few applications to image restoration problems.

\subsection{Image restoration examples}

\subsubsection{A gray-scale image}

For the following examples we used a two dimensional convolution model for the blurring operator with PSF of atmospheric turbulence type (gaussian kernel) with vertical and horizontal variances $\sigma_{v}^{2}$ and 
$\sigma_{h}^{2}$, respectively:

$$
\mathscr{T} w(x, y)=\iint_{\Omega}\left(2 \pi \sigma_{h} \sigma_{v}\right)^{-1} \exp \left(-\frac{(x-s)^{2}}{2 \sigma_{h}^{2}}-\frac{(y-t)^{2}}{2 \sigma_{v}^{2}}\right) w(s, t) d s d t
$$

Here $w(s, t)$, for $(s, t) \in \Omega \subset \mathbb{R}^{2}$, represents the gray-scale intensity at the point $(s, t)$ of the image we want to restore. The set $\Omega$ is the support of the image, which in all cases we took to be $[0,1] \times[0,1]$. Here too, model (28) was discretized in the usual way by taking a regular $M \times M$ grid on $\Omega$ and stacking the columns of the discretized version of the image $w(s, t)$ to form a vector $u \in \mathbb{R}^{M^{2}}$. The resulting discretized model was then of the form $T u=v$ where $T$ is an $M^{2}$-by- $M^{2}$ matrix and the components of $v$ correspond to the values of the observation $\mathscr{T} w(x, y)$ at the centerpoints of the corresponding pixels. For the examples that follow we took $\sigma_{v}^{2}=\sigma_{h}^{2}=0.02, M=100$ and the data of the inverse problem (namely $v$ ) was contaminated with $1 \%$ additive white noise.

Figure 2(a) shows the blurred-noisy image (data of the inverse problem) while Figure 2(b) shows the restoration obtained with a Tikhonov-Phillips method (pure $L^{2}$ penalizer). This restoration was later used to build the anisotropic penalization matrices $A_{m}$ (as done in [15]) and the components $\theta_{m}$ of the convex combination vector, in a similar way as it was done for signals.

(a)

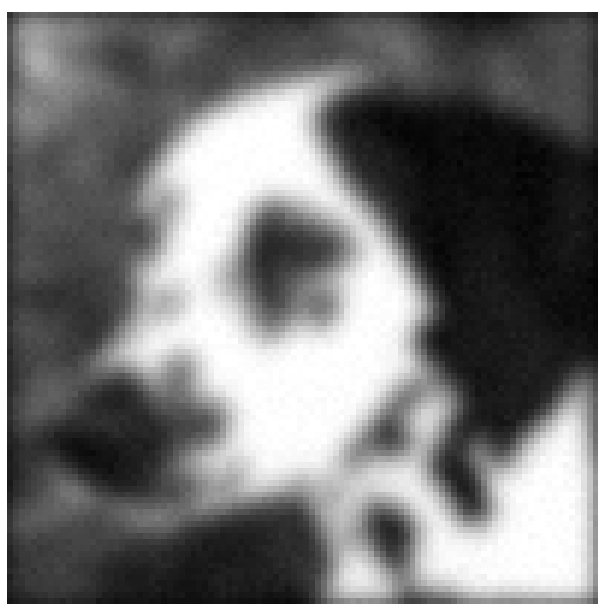

(b)

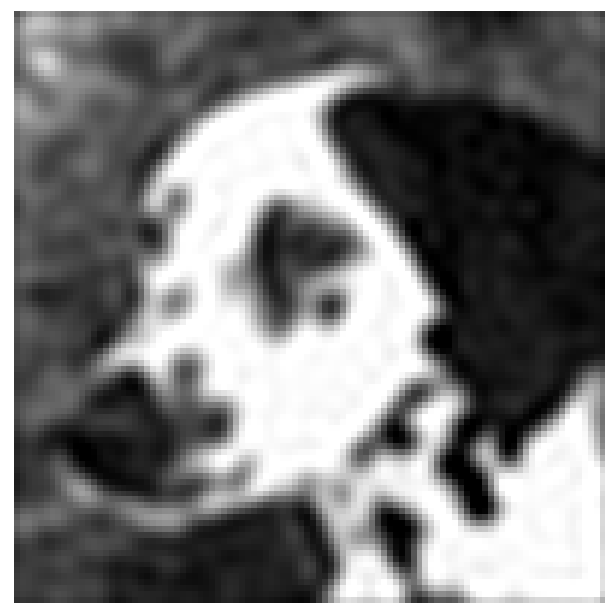

Figure 2: (a) Blurred noisy image (observation); (b) Tikhonov-Phillips restoration.

In Figure 3 we present the restorations obtained using pure $B V$ penalizers; isotropic case in (a) and anisotropic in (b). 
(a)

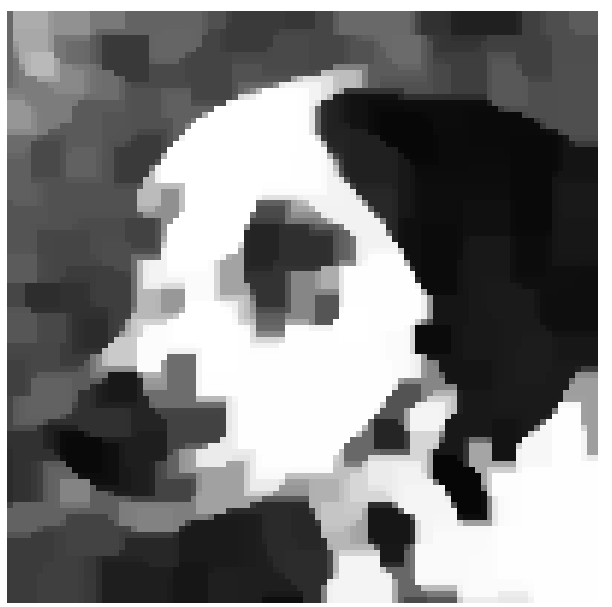

(b)

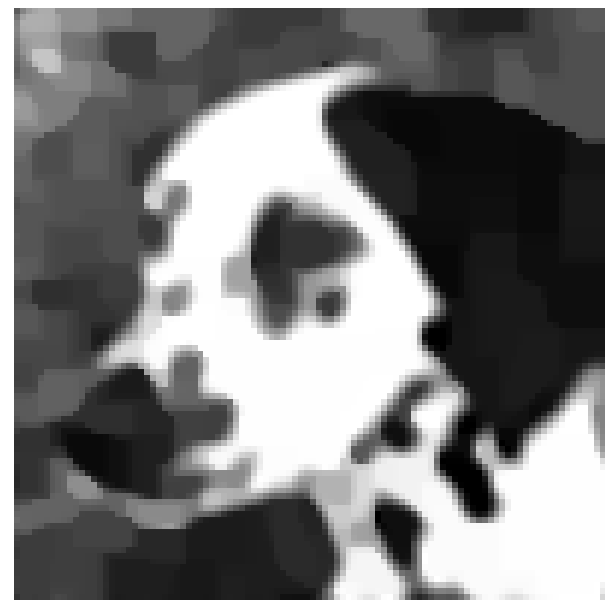

Figure 3: (a) Isotropic $B V$ restoration; (b) Anisotropic $B V$ restoration.

Finally, Figure 4 shows the restorations obtained with the mixed $L^{2}-B V$ penalizer; isotropic case in (a) and anisotropic in (b).

(a)

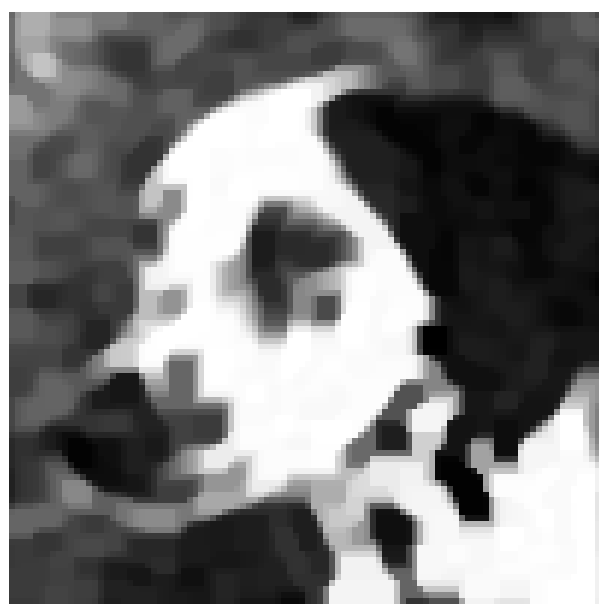

(b)

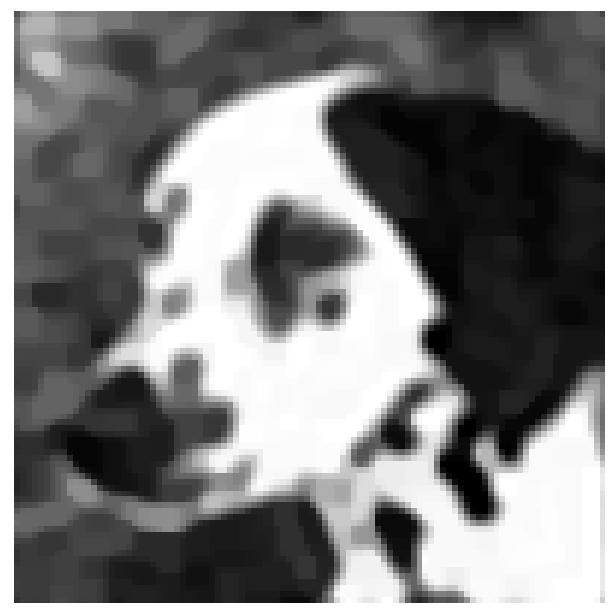

Figure 4: (a) Mixed $L^{2}$-isotropic $B V$ restoration; (b) Mixed $L^{2}$-anisotropic $B V$ restoration.

For comparison purposes, the original image is presented in Figure 5 and all ISNR values in Table 2. Once again, the best restoration is obtained with the combined $L^{2}$-anisotropic $B V$ penalizer. It is also timely to see that the ISNR value of the anistropic $B V$ restoration is significantly larger than the one obtained with the isotropic $B V$ penalizer. Also, the ISNR increases from any one of the single methods to the corresponding combined one (namely, isotropic $B V$ to mixed isotropic and anisotropic $B V$ to mixed anisotropic). These observations clearly highlight the relevance and potential applications of the combined methods. 


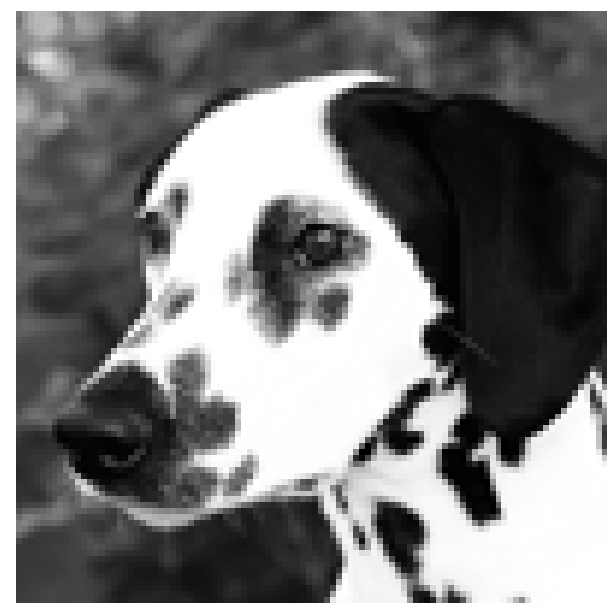

Figure 5: Original image

\begin{tabular}{lr} 
Restoration Type & ISNR \\
\hline Tikhonov & 4.140 \\
Isotropic BV & 3.666 \\
Anisotropic BV & 5.032 \\
Mixed Isotropic & 4.517 \\
Mixed Anisotropic & 5.118
\end{tabular}

Table 1: ISNRs of each restoration

\subsubsection{A color image}

Finally we present an application to a color image restoration problem. Here the forward model consists of applying the integral operator given by equation (28) to each one of the three layers (RGB) of the image. Blurring, noise contamination and restoration were all performed separately on each one of the layers. Figure 6(a) shows the blurred-noisy image (data) while Figure 6(b) shows the restoration obtained with a Tikhonov-Phillips (pure $L^{2}$ ) regularization method. Here too this restoration was used to build the anisotropic penalization matrices $A_{m}$ and the components $\theta_{m}$ of the convex combination vector.

Figure 7 shows the restorations obtained with pure $B V$ penalization terms, both isotropic (a) and anisotropic (b). The difference between the restorations induced by taking into account gradient-induced directions stands out clearly. Finally, Figure 8 shows the restorations performed with mixed $L^{2}-B V$ regularization. 
(a)

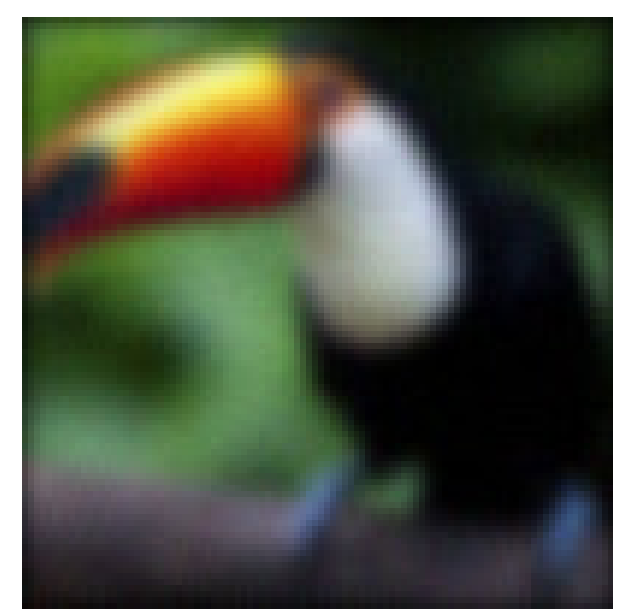

(b)

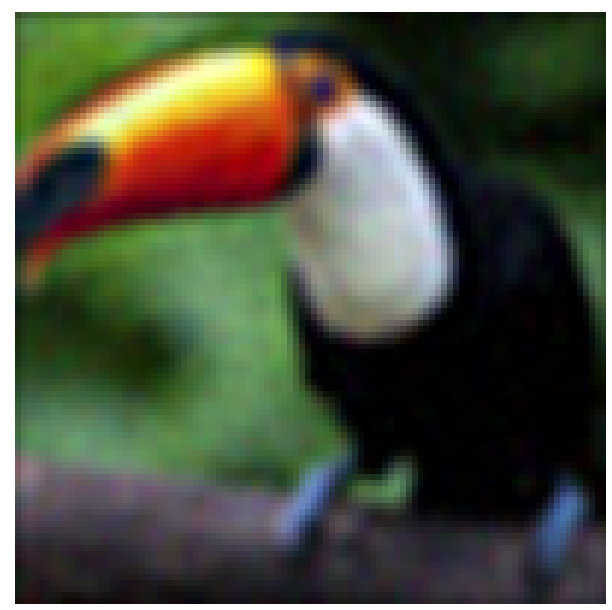

Figure 6: (a) Blurred noisy image (observation); (b) Tikhonov-Phillips restoration.

(a)

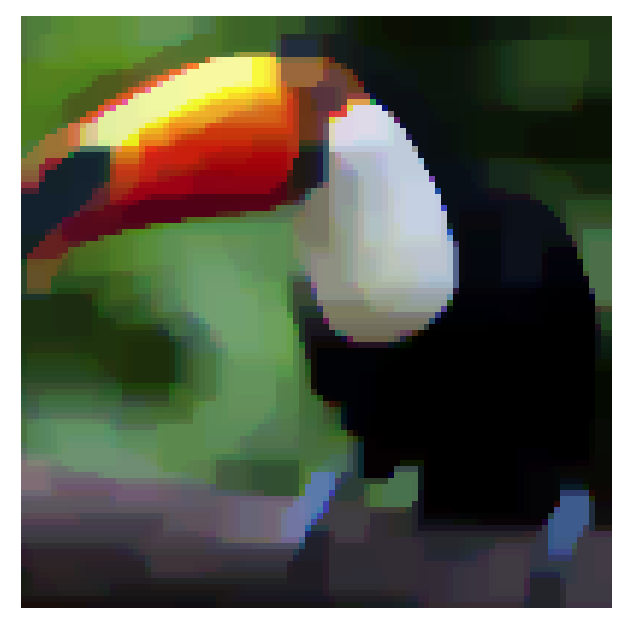

(b)

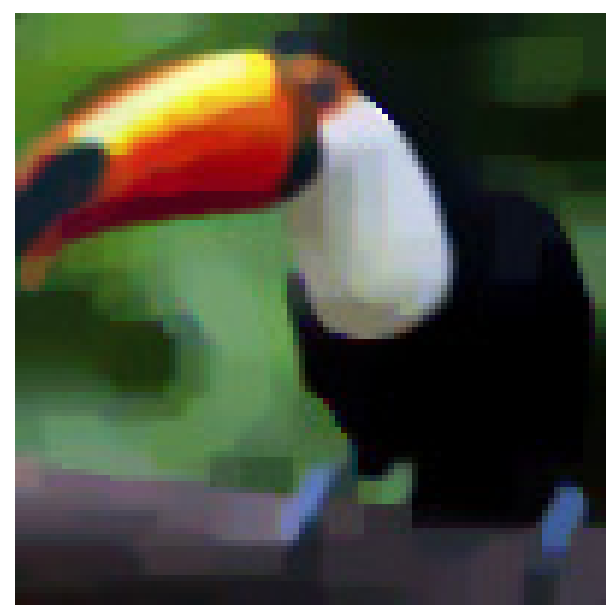

Figure 7: (a) Isotropic $B V$ restoration; (b) Anisotropic $B V$ restoration. 
(a)

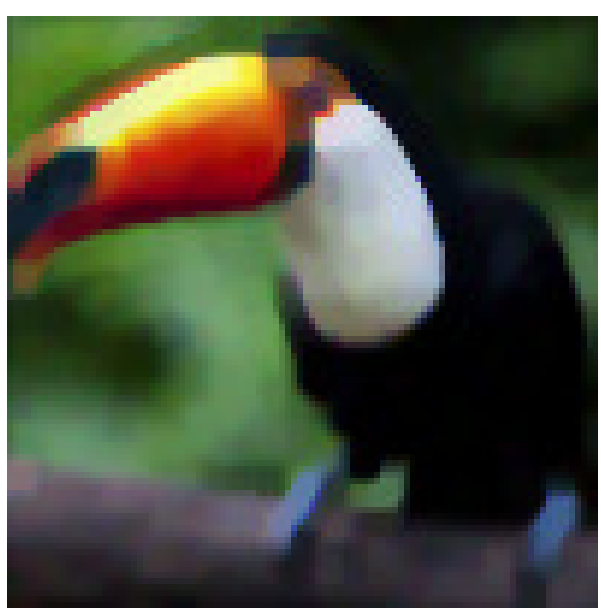

(b)

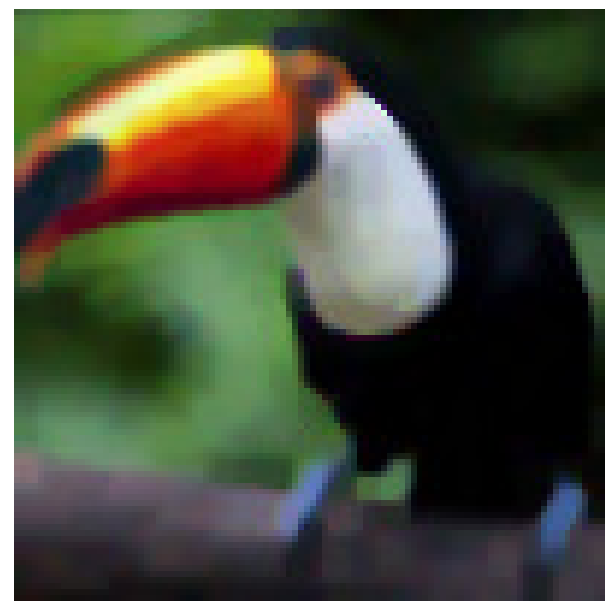

Figure 8: (a) Mixed $L^{2}$-isotropic $B V$ restoration; (b) Mixed $L^{2}$-anisotropic $B V$ restoration.

To better illustrate and compare the performances of the methods, Figure 9 shows the original image while Table 2 shows the ISNR values for each one of the five restorations.

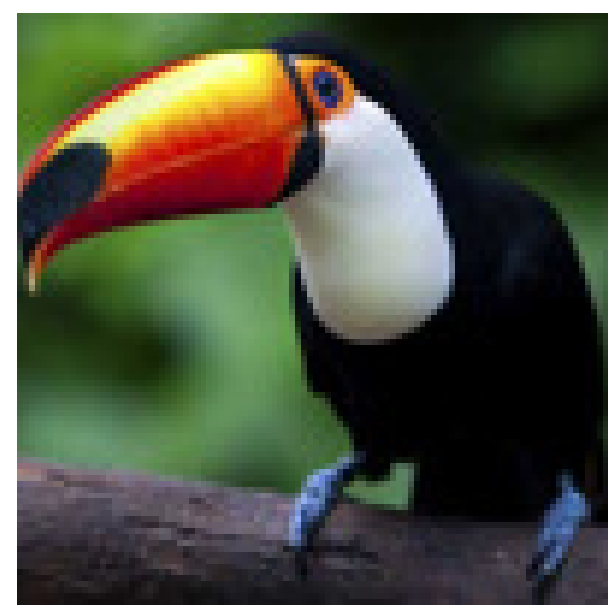

Figure 9: Original image

\begin{tabular}{ll} 
Restoration Type & ISNR \\
\hline Tikhonov & 3.325 \\
Isotropic BV & 3.104 \\
Anisotropic BV & 4.042 \\
Mixed Isotropic & 3.846 \\
Mixed Anisotropic & 4.420
\end{tabular}

Table 2: ISNRs of each restoration

Similar observations as for the gray-scale example can be made here. Namely, the best restoration is once again obtained with the combined $L^{2}$-anisotropic $B V$ penalizer, the ISNR value of the anisotropic $B V$ restoration is considerably larger than the one for the isotropic $B V$ penalizer and the ISNR values increase from any one of the single methods to the corresponding combined one. 
5. Conclusions

In this article we used a non-convex reformulation plus a non-quadratic half-quadratc approach to attack a general Tikhonov-Phillips regularization method where the penalizers are given by mixed spatially varying weighted convex combinations of $L^{2}$ and $B V$ functionals. Both isotropic and anisotropic $B V$ diffusion were considered. The associated optimization problems were then recast by means of a duality argument as half-quadratic optimization problems. The method proposed in this article resulted in significantly faster algorithms as compared to direct Newton-based methods. Numerical results in signal and image restoration problems were shown along with their ISNRs, whose higher values for the mixed-anisotropic restorations showed substantial improvements.

\section{Acknowledgements}

This work was supported in part by Agencia Nacional de Promoción Científica y Tecnológica, ANPCyT, through project PICT 2008-1301, by Consejo Nacional de Investigaciones Científicas y Técnicas, CONICET, through PIP 2010-2012 Nro. 0219, by Universidad Nacional del Litoral, through projects CAI+D PJov 2011 Nro. 50020110100055, CAI+D PI 2011 Nro. 50120110100294 and by the Air Force Office of Scientific Research, AFOSR, through Grant FA9550-14-1-0130.

\section{References}

[1] J. Hadamard, "Sur les problmes aux drives partielles et leur signification physique," Princeton University Bulletin, vol. 13, pp. 49-52, 1902.

[2] R. D. Spies and K. G. Temperini, "Arbitrary divergence speed of the least-squares method in infinite-dimensional inverse ill-posed problems," Inverse Problems, vol. 22, pp. 611-626, 2006.

[3] H. W. Engl, M. Hanke, and A. Neubauer, Regularization of inverse problems, vol. 375 of Mathematics and its Applications. Dordrecht: Kluwer Academic Publishers Group, 1996.

[4] D. L. Phillips, "A technique for the numerical solution of certain integral equations of the first kind," J. Assoc. Comput. Mach., vol. 9, pp. 84-97, 1962.

[5] A. N. Tikhonov, "Solution of incorrectly formulated problems and the regularization method," Soviet Math. Dokl., vol. 4, pp. 1035-1038, 1963.

[6] R. Acar and C. R. Vogel, "Analysis of bounded variation penalty methods for ill-posed problems," Inverse Problems, vol. 10, pp. 1217-1229, 1994.

[7] L. I. Rudin, S. Osher, and F. E., "Nonlinear total variation based noise removal algorithms (proceedings of the 11th annual international conference of the center for nonlinear studies)," Physica D, vol. 60, pp. 259-268, 1992.

[8] A. Chambolle and J. L. Lions, "Image recovery via total variation minimization and related problems," Numer. Math., vol. 76, pp. 167-188, 1997.

[9] G. L. Mazzieri, R. D. Spies, and K. G. Temperini, "Existence, Uniqueness and Stability of Minimizers of Generalized Tikhonov-Phillips Functionals," Journal of Mathematical Analysis and Applications, vol. 396, pp. 396-411, 2012.

[10] P. Blomgren, T. F. Chan, P. Mulet, and C. Wong, "Total variation image restoration: Numerical methods and extensions," Proceedings of the IEEE International Conference on Image Processing, 
vol. III, pp. 384-387, 1997.

[11] Y. Chen, S. Levine, and M. Rao, "Variable exponent, linear growth functionals in image restoration," SIAM Journal Applied on Mathematics, vol. 66, no. 4, pp. 1383-1406, 2006.

[12] F. Li, Z. Li, and L. Pi, "Variable exponent functionals in image restoration," Applied Mathematics and Computation, vol. 216, pp. 870-882, 2010.

[13] G. L. Mazzieri, R. D. Spies, and K. G. Temperini, "Mixed spatially varying $L^{2}-B V$ regularization of inverse ill-posed problems," Journal of Inverse and Ill-posed Problems (submitted 2014) ArXiv:1403.5579v1 [math.FA] 21 Mar 2014, http://arxiv.org/abs/1403.5579v1, 2014.

[14] G. L. Mazzieri, R. D. Spies, and K. G. Temperini, "Regularization of inverse ill-posed problems by anisotropic $L^{2}-B V$ penalization," In preparation.

[15] D. Calvetti, F. Sgallari, and E. Somersalo, "Image inpainting with structural boostrap priors," Image and Vision Computing, vol. 24, pp. 782-793, 2006.

[16] D. Geman and C. Yang, "Nonlinear image recovery with half-quadratic regularization," IEEE Transaction on Image Processing, Aachen, vol. 4, no. 7, pp. 932-946, 1995.

[17] J. Idier, Bayesian Approach to Inverse Problems. John Wiley \& Sons, 2008.

[18] R. Rockafellar, Convex Analysis. Princeton University Press, 1970. 